\title{
Aberrant expression of IL-23/IL-23R in patients with breast cancer and its clinical significance
}

\author{
SHUHAI SHENG ${ }^{1}$, JINJI ZHANG ${ }^{2}$, JIANZHONG AI ${ }^{3}$, XUELI HAO ${ }^{4}$ and RUISHEN LUAN ${ }^{4}$ \\ ${ }^{1}$ Department of Breast Cancer Surgery, Tangshan Gongren Hospital; ${ }^{2}$ North China University of Science and Technology \\ Affiliated Hospital; ${ }^{3}$ Department of Oncological Surgery, Tangshan Gongren Hospital, Tangshan, Hebei 063000; \\ ${ }^{4}$ Department of Breast and Neck Surgery, The Third Hospital of Chengde City, Chengde, Hebei 067000, P.R. China
}

Received July 11, 2017; Accepted December 21, 2017

DOI: $10.3892 / \mathrm{mmr} .2018 .8427$

\begin{abstract}
Breast cancer tissues and adjacent tissues were collected from 32 patients who were treated in The Third Hospital of Chengde City. Reverse transcription-quantitative polymerase chain reaction results demonstrated that, compared with the adjacent tissues, interleukin (IL)-23/IL-23 receptor $(\mathrm{R})$ gene expression levels were notably higher in breast cancer tissues. Furthermore, IL-23 and IL-23R expression levels were positively correlated with patients' tumor size, TNM stage and metastasis. Recombinant human (rh) IL-23 (10 $\mathrm{ng} / \mathrm{ml})$ was used for the stimulation of the MCF-7 cell line. Effects of rh IL-23 (10 ng/ml) on cell proliferation was detected after MCF-7 cells were incubated with rh IL-23 for $48 \mathrm{~h}$. Whether pre-treatment with polyclonal antibody (PAb) IL-23p19, a neutralizing antibody specific for IL-23, may influence the effects of IL-23 on cell behavior was also investigated. Cell proliferation assay and cell apoptosis assay were evaluated using MTT assay and flow cytometry assay, respectively. Results suggested that PAb IL-23p19 reduced IL-23-induced cell proliferation whereas induced IL-23 inhibited cell apoptosis. Western blot analysis was performed for the detection of molecules that may be responsible for the aforementioned changes. Results indicated that PAb IL-23p19 treatment reduced IL-23-induced upregulation of B-cell lymphoma-2 protein expression and activation of the janus kinase 2/signal transducer and activator of transcription 3 signaling pathway. The present results suggested that IL-23 may be a potential prognosis marker and target for the treatment of breast cancer patients.
\end{abstract}

Correspondence to: Dr Ruishen Luan, Department of Breast and Neck Surgery, The Third Hospital of Chengde City, 28 Puning North Road, Chengde, Hebei 067000, P.R. China

E-mail: luanruishencd@sina.com

Key words: interleukin-23, breast cancer, janus kinase 2/signal transducer and activator of transcription pathway

\section{Introduction}

Globally, breast cancer (BC) is the most frequently diagnosed cancer in women and accounts for more than 1 million new cases yearly (1). Despite the mortality of breast cancer has been reduced dueing to the early detection and adjuvant treatments, more than 450,000 deaths still occur each year (2). As acknowledged, breast cancer is a complex and heterogeneous disease which is accompanied with dissimilar cellular origin, etiology, treatment responses or clinical outcomes (3). Current therapies for breast cancer which are customized are based on molecular profiles including luminal, basal-like and human epidermal growth factor receptor-2 positive, and contain chemotherapy, endocrine therapy, selective estrogen receptor modulators and targeted therapies (4).

Interleukin (IL)-23 is a pro-inflammatory cytokine which consists of IL-12 p40 and IL-23 p19 subunits. IL-23, which is primarily secreted by macrophages and dendritic cells, induces autoimmunity by T-cell-mediated inflammation through impacting $\mathrm{T}$ helper 17 (Th17) cell response (5). Inappropriate expression of IL-23 appeared to coincide with multiple autoimmune disorders $(6,7)$. Furthermore, previous studies indicate that IL-23/IL-23R is indispensable for Th17 cell-mediated immune response $(8,9)$, and IL-23R plays a crucial role in initiating, maintaining and accelerating IL-23/IL-17 inflammatory signaling pathway (10). The importance of IL-23R during the development of tumor and the effects on tumor immunity was previously elucidated (11); meanwhile, IL-23R was indicated to exert an immunosurveillance function by $\mathrm{CD} 8^{+}$ T-cells and accelerate tumor growth in a previous study (12).

The correlation between chronic inflammation and elevated malignancy incidence or the regulatory mechanisms has been suggested for more than a century (13). The current study aimed to explore the effects of IL-23/IL-23R on breast cancer, and provide a therapeutic target for breast cancer.

\section{Materials and methods}

Participants. Breast cancer tissues and the adjacent tissues were gathered from thirty-two patients who were treated in our hospital. Basic characteristics of patients were presented in Table I. Prior written and informed consent were obtained from each patient before the beginning of our research. Current 
research was approved by the Ethics Committee of The Third Hospital of Chengde City.

Cell culture. MCF7 cells were maintained in Dulbecco's modified Eagle's medium (DMEM)/Ham's F12 medium with $10 \%$ fetal bovine serum, $100 \mathrm{U} / \mathrm{ml}$ penicillin and $100 \mu \mathrm{g} / \mathrm{ml}$ streptomycin. And incubated in a humidified atmosphere of $5 \% \mathrm{CO}_{2}$ at $37^{\circ} \mathrm{C}$.

Recombinant human (rh) IL-23 (R\&D) was used for the stimulation of the cells. Effects of rh IL-23 (10 ng/ml) on cell proliferation were detected after cells were incubated with it $48 \mathrm{~h}$; the influence of pre-treatment of PAb IL-23p19 (Santa Cruz Biotechnology, Inc., Dallas, TX, USA), a neutralizing antibody specific for IL-23, on rh IL-23 treated cells were also examined (14). Therefore, cells were divided into 3 groups, i.e., control group, IL-23 group (cells treated with rh IL-23) and PAb IL-23p19 group (cells treated with PAb IL-23p19 and rh IL-23) to investigate cell behaviors and correlated protein expression levels.

Real-time PCR analysis of IL-23R messenger RNA. Tumor tissues and the adjacent tissues of thirty-two breast cancer patients were extracted and stored at $-80^{\circ} \mathrm{C}$ or applied for the following experiments. Total RNA was isolated by TRIzol (Molecular Research Center, Cincinnati, OH, USA). cDNA was obtained from mRNA with oligo primers and Superscript II (Invitrogen; Thermo Fisher Scientific, Inc., Waltham, MA, USA) based on the manufacturer's protocol. Relative gene level for IL-23R was quantified by ABI Prism 7000 (Applied Biosystems; Thermo Fisher Scientific, Inc., Waltham, MA, USA) based on the method of SYBR-Green. Primers were as followed: IL-23, forward: TGCTAGGATCGGATATTT TCACAGG; reverse: GAGGCTTGGAATCTGCTGAGT C; IL-23R, forward: GATATTCCTGATGAAGTAACCTGT GT. C; reverse: GATACTGTTGCTCTTCTTCTGTCTC; GAPDH, forward: CAGCCTCAAGATCATCAGCA; reverse: ACAGTCTTCTGGGTGGCAGT, which were as previously reported study performed (14). Total volume of PCR reaction was $20 \mu \mathrm{l}$ which consisted of $0.1 \mu \mathrm{M}$ forward/reverse primer, 1x SYBR Premix EX Taq premix (Takara Biotechnology Co., Ltd., Dalian, China) and 50 ng cDNA. The conditions were: $95^{\circ} \mathrm{C}$ for $120 \mathrm{sec}$, followed by 40 cycles at $95^{\circ} \mathrm{C}$ for $15 \mathrm{sec}$ and $60^{\circ} \mathrm{C}$ for $60 \mathrm{sec}$. GAPDH was used as an internal reference gene.

Immunological histological chemistry. Tissues from patients were first sliced into $4 \mathrm{~mm}$-thick sections. Afterwards, sections were fixed by $7.5 \%$ buffered formalin and embedded with paraffin. Then deparaffinage and antigen retrieval of slices were performed before incubation with antibody. Avidin-biotin-peroxidase complex (ABC) method was conducted with monoclonal IL-23 antibody and IL-23R antibody according to the manufacturer's protocol. The expression levels of IL-23 and IL-23R were independently assessed by three senior pathologists in accordance with the proportion and intensity of positive cells.

MTT assay. Cell proliferation was examined by MTT assay. Briefly, cells $\left(1 \times 10^{5}\right)$ were seeded into 12 -well plate and incubated with methamphetamine for $24 \mathrm{~h}$. MTT ( $5 \mathrm{mg} / \mathrm{ml}$ in PBS) was added into the wells, and cells were incubated for another $1.5 \mathrm{~h}$ at $37^{\circ} \mathrm{C}$. After extraction and throwing away the supernatant from the culture medium, dimethylsulfoxide $(200 \mu \mathrm{l})$ was added to dissolve the formazan crystals. The optical density (OD) on a spectrophotometer was measured at $595 \mathrm{~nm}$.

Cell apoptosis analysis. Cells were seeded in 12-well plates and cultured for $48 \mathrm{~h}$ in an incubator at $37^{\circ} \mathrm{C}$ with humidified atmosphere of $5 \% \mathrm{CO}_{2}$. A total of $1 \times 10^{5}$ cells were collected by centrifugation $(2,000 \mathrm{rpm}, 5 \mathrm{~min})$ and washed by ice-cold PBS. Afterwards, cells were fixed with ice-cold ethanol (70\%) and placed at $-20^{\circ} \mathrm{C}$ overnight. On the next day, cells were stained by Annexin $\mathrm{V}$-enhanced green fluorescent protein (FITC) and propidium iodide (PI), then incubated for $15 \mathrm{~min}$ at room temperature in dark place. Assay results were measured by Cell Quest software (BD Biosciences, Franklin Lakes, NJ, USA). The percentage of cells with apoptotic nuclei was calculated.

Western blot analysis. Cell were rinsed twice with ice cold PBS and scraped by RIPA (Beyotime Institute of Biotechnology, Shanghai, China). Samples were separated by $10 \%$ sodium dodecyl sulfate polyacrylamide gel electrophoresis. Then, gels were transferred onto polyvinylidene difluoride membranes at $4^{\circ} \mathrm{C}$. Membranes reacted with primary antibody overnight at $4^{\circ} \mathrm{C}$, and horseradish peroxidase-conjugated secondary antibody for $90 \mathrm{~min}$ at room temperature with gentle agitation. Finally, at room temperature, after washing with Tris Buffered Saline with Tween 20 (TBST) for $10 \mathrm{~min}$, proteins were detected with Super Signal West Pico Chemiluminescent Substrate (Thermo Fisher Scientific, Inc.).

Statistical analysis. Data were analyzed by SPSS v21.0 (SPSS, Inc., Chicago, IL, USA) and presented as mean \pm standard error of mean (SEM). Data were analyzed by t-test. $\mathrm{P}<0.05$ was considered to indicate a statistically significant difference.

\section{Results}

mRNA level of IL-23/IL-23R is higher in breast cancer tissues than the adjacent tissues. Previous study has reported the differences of IL-23 mRNA expression level between tumor tissues and the adjacent normal tissues in numerous types of organs, including conlon, ovarian, lung, stomach and breast, among all of which there was significant up-regulation of IL-23 mRNA level in tumor tissues than in the normal tissues (12). In our present study, we also first explored the differences of IL-23 mRNA level between breast cancer tissue and the adjacent normal tissue by RT-PCR. Results indicated that, IL-23 mRNA level was also significantly higher in tumor tissue in comparison with the normal tissue $(\mathrm{P}<0.01, \mathrm{n}=32$, Fig. 1A), which was in consistent with the previous reported study (12). Moreover, IL-23 was considered as a prognostic factor in breast cancer patients which was reported by Gangemi et al (15). Therefore, we put forward a hypothesis that IL-23-mediated responses might be crucial for the promotion of tumor.

Studies found that genetic variants of IL-23R might contribute to the pathological development of hepatitis to HCC (16); while hepatitis B virus could induce hepatitis by elevating IL-23 expression and lead to liver damage via 
Table I. IL-23/IL-23R mRNA level was correlated with clinicopathological parameters of breast cancer patients.

\begin{tabular}{|c|c|c|c|c|c|}
\hline Parameters & Cases & IL-23 & P-value & IL-23R & P-value \\
\hline Age (years) & & & 0.71 & & 0.54 \\
\hline$<40$ & 15 & $5.21 \pm 0.67$ & & $48.60 \pm 8.04$ & \\
\hline$\geq 40$ & 17 & $5.79 \pm 0.74$ & & $52.29 \pm 8.97$ & \\
\hline Tumor size (cm) & & & $0.04^{\mathrm{a}}$ & & $0.02^{\mathrm{a}}$ \\
\hline$\geq 5$ & 13 & $5.07 \pm 0.72$ & & $48.75 \pm 5.97$ & \\
\hline$<5$ & 19 & $5.74 \pm 0.94$ & & $54.14 \pm 6.01$ & \\
\hline Histological grade & & & 0.55 & & 0.78 \\
\hline Well-intermediately differentiation & 11 & $5.36 \pm 0.87$ & & $51.35 \pm 5.90$ & \\
\hline Poor differentiation & 21 & $5.14 \pm 1.02$ & & $51.98 \pm 5.95$ & \\
\hline Invasion depth & & & 0.78 & & 0.93 \\
\hline $\mathrm{T} 1-\mathrm{T} 2$ & 25 & $5.06 \pm 0.90$ & & $51.78 \pm 5.90$ & \\
\hline T3-T4 & 7 & $5.24 \pm 1.06$ & & $51.99 \pm 6.02$ & \\
\hline Lymph node metastasis & & & 0.89 & & 0.72 \\
\hline No & 3 & $5.17 \pm 0.90$ & & $50.87 \pm 5.90$ & \\
\hline $\mathrm{N} 1-\mathrm{N} 3$ & 29 & $5.08 \pm 1.06$ & & $51.98 \pm 4.96$ & \\
\hline Distant metastasis & & & $0.04^{\mathrm{a}}$ & & $0.02^{\mathrm{a}}$ \\
\hline M0 & 27 & $5.02 \pm 0.68$ & & $50.06 \pm 5.99$ & \\
\hline M1 & 5 & $5.75 \pm 0.71$ & & $57.17 \pm 4.76$ & \\
\hline TNM stage & & & $0.01^{\mathrm{a}}$ & & $0.04^{\mathrm{a}}$ \\
\hline 0 -II & 23 & $5.05 \pm 0.74$ & & $50.17 \pm 5.90$ & \\
\hline III- IV & 9 & $5.88 \pm 0.66$ & & $56.14 \pm 5.76$ & \\
\hline
\end{tabular}

${ }^{\mathrm{a}} \mathrm{P}<0.05$. IL-23R, interleuking-23 receptor.

IL-23/IL-17 axis (17). IL-23R plays a crucial role in initiating, maintaining and accelerating IL-23/IL-17 inflammatory signaling pathway (10). Since IL-23 mRNA level was upregulated in breast cancer tissue than in the adjacent normal tissue, we were eager to know the changes of IL-23R between tumor tissue and the normal tissue. RT-PCR results exhibited that, there was much higher mRNA level of IL-23R in breast cancer tissue compared to the normal tissue $(\mathrm{P}<0.001, \mathrm{n}=32$, Fig. 1B).

Meanwhile, we detected the protein levels of IL-23 and IL-23R in the adjacent tissues and tumor tissues from patients by immunohistochemistry. We found that, both IL-23 and IL-23R exhibited much more expression level in tumor tissues in comparison with the adjacent tissues (Fig. 1C-F).

Correlation between IL-23/IL-23R and patients' characteristics. Both IL-23 and IL-23R were positively correlated with patients' tumor size, TNM stage and metastasis. Patients were divided into two groups according to the age, there were 17 patients aged $\geq 40$ and 15 patients aged $<40$, IL-23 and IL-23R in patients aged $\geq 40$ years old were higher than in patients aged $<40$ years old, however, there was no significant difference; as for histological grade, there were 11 patients showed well-intermediately differentiation while 21 patients showed poor differentiation, IL-23/IL-23R in poor differentiation was a little lower than in well-intermediately differentiation, whereas, there was no significant difference; regarding invasion depth, there were 25 patients at T1-T2 and 7 patients at T3-T4, we found that, IL-23/IL-23R levels were higher in T3-T4 group than in T1-T2 group, however, there was no significant difference (Table I).

Pre-treatment of PAb IL-23p19 repressed IL-23 induced cell proliferation. To investigate whether IL-23 could induce cell proliferation of breast cancer cells, cell proliferation assay was performed. Cell proliferation rate revealed to be distinctly increased by treatment with $10 \mathrm{ng} / \mathrm{ml} \mathrm{rhIL}-23$ in comparison with the untreated control $(\mathrm{P}<0.001, \mathrm{n}=3$, Fig. $2 \mathrm{~A}$ and $\mathrm{B})$. Cells were further treated with IL-23 at the presence of PAb IL-23p19, PAb IL-23p19 abolished the induction of cell proliferation by IL-23 $(\mathrm{P}<0.01, \mathrm{n}=3$, Fig. $2 \mathrm{~A}$ and $\mathrm{B})$. Taken together, these results demonstrated that, the promotion of cell proliferation was an effect of IL-23 in breast cancer cell line, which could be distinctly inhibited by the pre-treatment of PAb IL-23p19.

Pre-treatment of PAb IL-23p19 induced IL-23 repressed cell proliferation. To investigate whether IL-23 could inhibit cell apoptosis of breast cancer cells, FCM assay was carried out. Cell apoptosis rate revealed to be distinctly decreased by $10 \mathrm{ng} / \mathrm{ml} \mathrm{rhIL}-23$ in comparison with control group $(\mathrm{P}<0.01$, $n=3$, Fig. $3 A$ and B). Cells were further treated with IL-23 at the presence of PAb IL-23p19, PAb IL-23p19 abolished the inhibition of cell apoptosis by IL-23 ( $\mathrm{P}<0.001, \mathrm{n}=3$, Fig. 3A and $\mathrm{B})$. Taken together, these results suggested that, the inhibition of cell apoptosis is also an effect of IL-23 in breast cancer cell line, which could be abolished by the pre-treatment of PAb IL-23p19. 
A
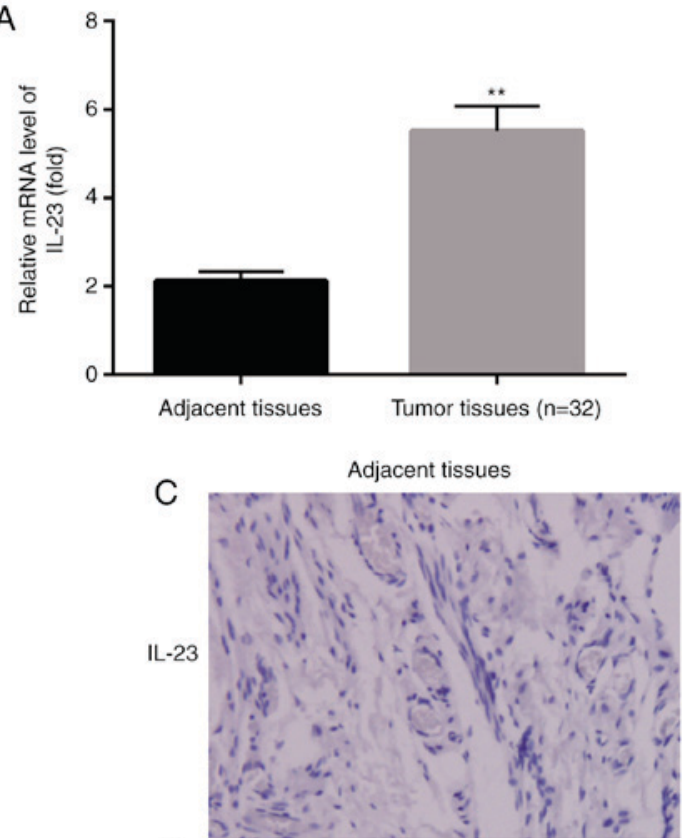

E

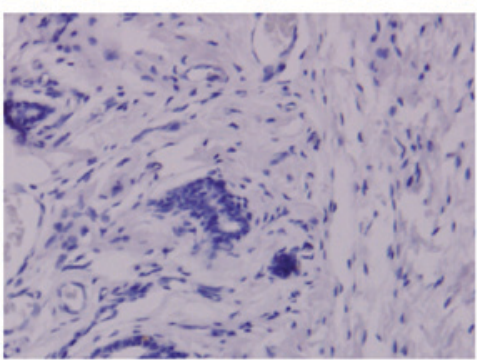

B

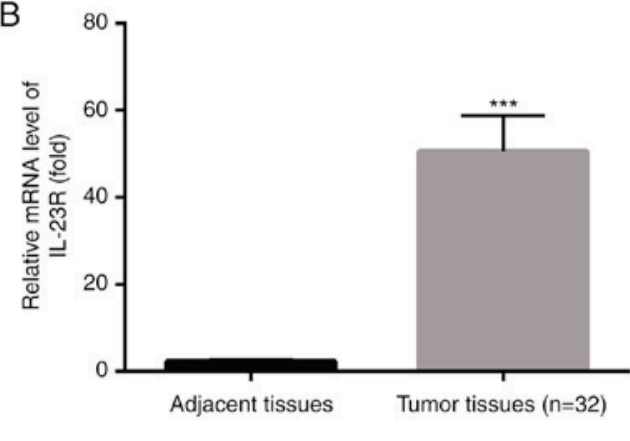

D

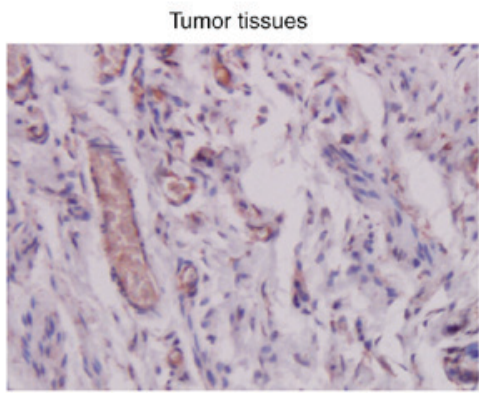

$\mathrm{F}$

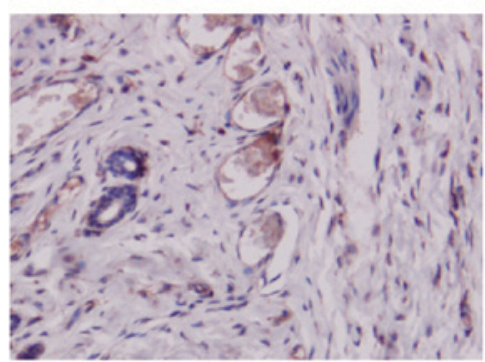

Figure 1. mRNA level and protein level of IL-23/IL-23R are higher in breast cancer tissues than the adjacent tissues. (A and B) We explored the differences of IL-23/IL-23R mRNA level between breast cancer tissue and the adjacent normal tissue by RT-PCR. Results indicated that, IL-23/IL-23R mRNA level was significantly higher in tumor tissue in comparison with the normal tissue. (C-F) Meanwhile, we detected the protein levels of IL-23/IL-23R in the adjacent tissues and tumor tissues by immunohistochemistry. Results revealed that, IL-23/IL-23R protein level was also significantly higher in tumor tissues than those in the adjacent tissues. ${ }^{* *} \mathrm{P}<0.01 ;{ }^{* * *} \mathrm{P}<0.001$, tumor tissues vs. adjacent tissues

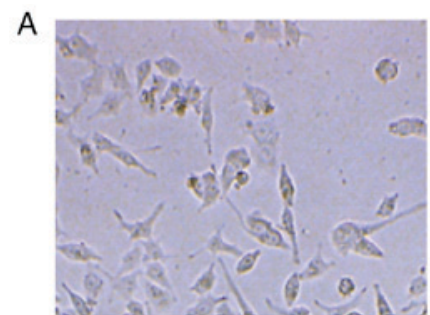

Control



IL-23

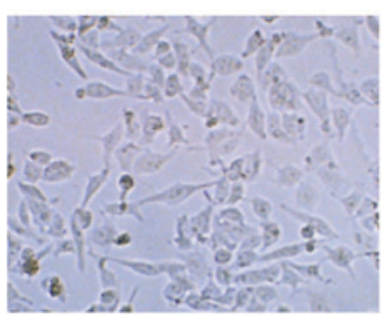

PAb IL-23p19

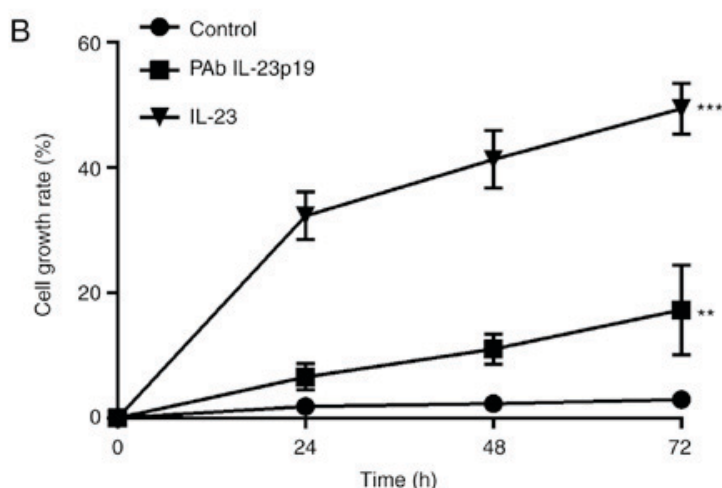

Figure 2. Pre-treatment of PAb IL-23p19 repressed IL-23 induced cell proliferation. (A and B) Cell proliferation of breast cancer cells was performed by cell proliferation assay. Cell proliferation rate was distinctly increased by $10 \mathrm{ng} / \mathrm{ml} \mathrm{rhIL-23} \mathrm{in} \mathrm{comparison} \mathrm{with} \mathrm{the} \mathrm{untreated} \mathrm{control,} \mathrm{PAb} \mathrm{IL-23p19} \mathrm{abolished} \mathrm{the}$ induction of cell proliferation induced by IL-23. ${ }^{* *} \mathrm{P}<0.01 \mathrm{PAb}$ IL-23p19 vs. IL-23 group; ${ }^{* * *} \mathrm{P}<0.001 \mathrm{IL}-23$ group vs. control group. 



Figure 3. (A and B) Pre-treatment of PAb IL-23p19 induced IL-23 repressed cell apoptosis. FCM assay was carried out to test cell apoptosis rate, which revealed to be distinctly decreased by $10 \mathrm{ng} / \mathrm{ml}$ rhIL-23 in comparison with control group, and PAb IL-23p19 abolished the inhibition of cell apoptosis by IL-23. ${ }^{* * *} \mathrm{P}<0.001 \mathrm{PAb}$ IL-23p19 vs. IL-23 group; ${ }^{* * *} \mathrm{P}<0.01$ IL-23 group vs. control group.

Pre-treatment of PAb IL-23p19 inhibited the activation of JAK2/STAT3 signaling pathway induced by IL-23. Recent evidence indicated that IL-23-mediated responses were crucial in promoting tumor progression of various tissues. IL-23 signaling occurred by activation of the JAK/STAT (specifically STAT3) pathway, thus resulting in up-regulation of proteins which were linked with proliferation, survival (bcl-x), metastasis (VEGF, HIF1 $\alpha$, MMP2, MMP9), angiogenesis and immunosuppression, etc (18). Consequently, we tested the expression levels of STAT3, JAK2 and bcl-2 in MCF-7 cells by western blot. Results showed that, the activation levels of STAT3 and JAK2 were induced by IL-23, and pre-treatment of PAb IL-23p19 inhibited the activation of STAT3 and JAK2. Meanwhile, bcl-2 protein expression was also upregulated by IL-23, which was downregulated by pre-treatment of PAb IL-23p19 (Fig. 4). These results suggested that, pre-treatment of PAb IL-23p19 abolished IL-23-mediated aberrant cell proliferation and apoptosis by inhibiting the activation of JAK2/STAT3 signaling pathway and reduced the expression of bcl-2.

\section{Discussion}

IL-23 was identified as a cancer-related cytokine in a recent study. The expression of IL-23 was significantly increased in majority of human tumors from various organs compared to normal adjacent tissues (12). As acknowledged, multiple cancers arose from chronic inflammation and inflammatory mediators were synthesized by tumors $(19,20)$. The ability to respond to IL-23 is determined by the expression level of IL-23R in cells (21).

In our study, we indicated that mRNA and protein levels of IL-23 and its receptor IL-23R were constitutively

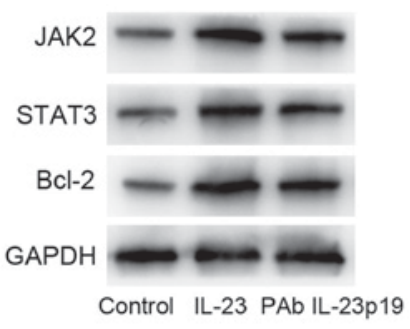

Figure 4. Pre-treatment of PAb IL-23p19 inhibited the activation of JAK2/STAT3 pathway induced by IL-23. We tested the expression levels of STAT3, JAK2 and bcl-2 in MCF-7 cells by western blot. Results showed that, the activation levels of STAT3 and JAK2 were induced by IL-23, and pre-treatment of PAb IL-23p19 inhibited the activation of STAT3 and JAK2. Meanwhile, bcl-2 protein expression was also upregulated by IL-23, which was downregulated by pre-treatment of PAb IL-23p19.

co-expressed in breast cancer tissues, and IL-23/IL-23R was increased in breast cancer tissues in comparison with the adjacent tissues (Fig. 1), which was in consistent with a previous reported research (12). Taken together, the results verified the potential of IL-23 as a prognosis factor in breast cancer.

Thereafter, we determined the effects of IL-23 on breast cancer cell line MCF-7 behaviors, and studied whether a neutralizing antibody specific for IL-23, PAb IL-23p19 could attenuate the effects of IL-23.

To investigate whether IL-23 could induce cell proliferation of breast cancer cells, cell proliferation assay was carried out. Cell proliferation rate revealed to be distinctly increased by treatment with rhIL-23 in comparison with control; cells were further treated with IL-23 at the presence of PAb IL-23p19, and PAb IL-23p19 abolished IL-23 induced cell proliferation (Fig. 2A and B). 
We also investigate whether IL-23 could inhibit cell apoptosis of breast cancer cells, FCM assay was carried out. Cell apoptosis rate revealed to be distinctly decreased by rhIL-23 in comparison with control; cells were further treated with IL-23 at the presence of PAb IL-23p19, PAb IL-23p19 abolished the inhibition of cell apoptosis by IL-23 (Fig. 3A and B).

Taken together, these results suggested that, the inhibition of cell apoptosis and promotion of cell proliferation by IL-23 could be abolished by pre-administration of PAb IL-23p19. Whereas, it was unclear which signaling pathway did IL-23 depend on to function.

IL-23-mediated responses were indicated to be crucial in promoting tumor progression. IL-23 signaling occurred by activating JAK/STAT3 pathway, up-regulating proteins that were correlated with proliferation and survival (bcl-x) (18). The JAK-STAT signaling cascade consisted of three main components: One Janus kinase (JAK, a cell surface receptor) and two signal transducer and activator of transcription (STAT) proteins; JAK-STAT signaling pathway transmitted information from extracellular chemical signals to nucleus, generating DNA transcription and expression of genes which were correlated with proliferation, apoptosis and oncogenesis (22). Disruption or dysregulation of JAK-STAT functionality lead to immune deficiency syndromes and cancers (22). Activated JAKs phosphorylated tyrosine residues on the receptor, creating binding sites for proteins which possessed SH2 domains; SH2 domain containing STATs were recruited to the receptor and tyrosine-phosphorylated (activated) by JAKs followed by forming hetero- or homodimers and translocating to the cell nucleus to induce target gene transcription (23). Moreover, activation of JAK2/STAT3 pathway contributed to the poor outcomes of metastatic breast cancers $(24,25)$.

Consequently, we tested the expression levels of STAT3, JAK 2 and bcl-2 in MCF-7 cells by western blot. Results showed that, the activation levels of STAT3 and JAK2 were induced by IL-23, and pre-treatment of PAb IL-23p19 inhibited the activation of STAT3 and JAK2. Meanwhile, bcl-2 protein expression was also upregulated by IL-23, which was downregulated by pre-treatment of PAb IL-23p19 (Fig. 4). These results suggested that, pre-treatment of PAb IL-23p19 abolished IL-23-mediated aberrant cell proliferation and apoptosis by inhibiting the activation of JAK2/STAT3 signaling pathway and reduced the expression of bcl-2.

In conclusion, blocking the function of IL-23 inhibited the proliferative activity and induced the apoptotic activity of tumor cells via inhibiting the activation of JAK2/STAT3 pathway in MCF-7 cells. We provide a potential therapeutic target for breast cancer.

\section{References}

1. Siegel R, Naishadham D and Jemal A: Cancer statistics, 2013. CA Cancer J Clin 63: 11-30, 2013.

2. Jemal A, Siegel R, Xu J and Ward E: Cancer statistics, 2010. Cancer J Clin 60: 277-300, 2010.

3. Cardoso F, Harbeck N, Barrios CH, Bergh J, Cortés J, EI Saghir N, Francis PA, Hudis CA, Ohno S, Partridge AH, et al: Research needs in breast cancer. Ann Oncol 28: 208-217, 2017.
4. Harbeck N and Gnant M: Breast cancer. Lancet 389: 1134-1150, 2017.

5. Ahern PP, Izcue A, Maloy KJ and Powrie F: The interleukin-23 axis in intestinal inflammation. Immunol Rev 226: 147-159, 2008.

6. Langrish CL, Chen Y, Blumenschein WM, Mattson J, Basham B, Sedgwick JD, McClanahan T, Kastelein RA and Cua DJ: IL-23 drives a pathogenic $\mathrm{T}$ cell population that induces autoimmune inflammation. J Exp Med 201: 233-240, 2005.

7. Lee E, Trepicchio WL, Oestreicher JL, Pittman D, Wang F, Chamian F, Dhodapkar M and Krueger JG: Increased expression of interleukin 23 p19 and p40 in lesional skin of patients with psoriasis vulgaris. J Exp Med 199: 125-130, 2004.

8. Chen Z, Laurence A and O'Shea JJ: Signal transduction pathways and transcriptional regulation in the control of Th17 differentiation. Semin Immunol 19: 400-408, 2007.

9. Volpe E, Servant N, Zollinger R, Bogiatzi SI, Hupé P, Barillot E and Soumelis V: A critical function for transforming growth factorbeta, interleukin 23 and proinflammatory cytokines in driving and modulating human $\mathrm{T}(\mathrm{H})-17$ responses. Nat Immunol 9: 650-657, 2008.

10. Cho JH and Weaver CT: The genetics of inflammatory bowel disease. Gastroenterology 133: 1327-1339, 2007.

11. Le Gouvello S, Bastuji-Garin S, Aloulou N, Mansour H, Chaumette MT, Berrehar F, Seikour A, Charachon A, Karoui M, Leroy K, et al: High prevalence of Foxp3 and IL17 in MMR proficient colorectal carcinomas. Gut 57: 772-779, 2008.

12. Langowski JL, Zhang X, Wu L, Mattson JD, Chen T, Smith K, Basham B, McClanahan T, Kastelein RA and Oft M: IL-23 promotes tumor incidence and growth. Nature 442: 461-465, 2006.

13. Balkwill $\mathrm{F}$ and Mantovani A: Inflammation and cancer: Back to Virchow? Lancet 357: 539-545, 2001.

14. Fukuda M, Ehara M, Suzuki S and Sakashita H: Expression of interleukin-23 and its receptors in human squamous cell carcinoma of the oral cavity. Mol Med Rep 3: 89-93, 2010.

15. Gangemi S, Minciullo P, Adamo B, Franchina T, Ricciardi GR, Ferraro M, Briguglio R, Toscano G, Saitta S and Adamo V: Clinical significance of circulating interleukin-23 as a prognostic factor in breast cancer patients. J Cell Biochem 113: 2122-2125, 2012.

16. Xu Y, Liu Y, Pan S, Liu L, Liu J, Zhai X, Shen H and Hu Z: IL-23R polymorphisms, HBV infection and risk of hepatocellular carcinoma in a high-risk Chinese population. J Gastroenterol 48: 125-131, 2013.

17. Wang Q, Zhou J, Zhang B, Tian Z, Tang J, Zheng Y, Huang Z, Tian Y, Jia Z, Tang Y, et al: Hepatitis B virus induces IL-23 production in antigen presenting cells and causes liver damage via the IL-23/IL-17 axis. PLoS Pathog 9: e1003410, 2013.

18. Yu H, Kortylewski M and Pardoll D: Crosstalk between cancer and immune cells: Role of STAT3 in the tumour microenvironment. Nat Rev Immunol 7: 41-51, 2007.

19. Coussens LM and Werb Z: Inflammation and cancer. Nature 420: 860-867, 2002

20. Balkwill F, Charles KA and Mantovani A: Smoldering and polarized inflammation in the initiation and promotion of malignant disease. Cancer Cell 7: 211-217, 2005.

21. Parham C, Chirica M, Timans J, Vaisberg E, Travis M, Cheung J, Pflanz S, Zhang R, Singh KP, Vega F, et al: A receptor for the heterodi-meric cytokine IL-23 is composed of IL-12Rbeta1 and a novel cytokine receptor subunit, IL-23R. J Immunol 168: 5699-5708, 2002.

22. Aaronson DS and Horvath CM: A road map for those who don't know JAK-STAT. Science 296: 1653-1655, 2002.

23. Hebenstreit D, Horejs-Hoeck J and Duschl A: JAK/STAT-dependent gene regulation by cytokines. Drug News Perspect 18: 243-249, 2005.

24. Marotta LL, Almendro V, Marusyk A, Shipitsin M, Schemme J, Walker SR, Bloushtain-Qimron N, Kim JJ, Choudhury SA, Maruyama R, et al: The JAK2/STAT3 signaling pathway is required for growth of $\mathrm{CD} 44^{+} \mathrm{CD} 24^{-}$stem cell-like breast cancer cells in human tumors. J Clin Invest 121: 2723-2735, 2011.

25. Hennessy BT, Gonzalez-Angulo AM, Stemke-Hale K, Gilcrease MZ, Krishnamurthy S, Lee JS, Fridlyand J, Sahin A, Agarwal R, Joy C, et al: Characterization of a naturally occurring breast cancer subset enriched in epithelial-to-mesenchymal transition and stem cell characteristics. Cancer Res 69: 4116-4124, 2009. 